\title{
Sobre la enseñanza de la economía
}

\author{
Laura Buitrago Niño y Enrique Ferrer-Corredor
}

Hemos entrevistado a cuatro académicos muy destacados en el ámbito universitario colombiano sobre la evolución y actualidad de la enseñanza de la economía. Recogemos en este apartado sus voces, a manera de un diálogo privilegiado que compartimos con la comunidad intelectual para enriquecer el debate, no solo alrededor el discurso económico, sino de paso como es de nuestro interés en esta entrega de la revista Papeles, en cofradía con las facultades de educación. Agradecemos al Dr. Mauricio Pérez Salazar, exdecano de la Facultad de Economía de la Universidad Externado de Colombia; al Dr. Erick Behar Villegas, decano de la Facultad de Economía de la Universidad Central; a la Dra. Juanita Villaveces Niño, directora de la Escuela de Economía de la Universidad Nacional; y al Dr. Carlos Julio Martínez Becerra, decano de la Facultad de Ciencias Económicas y Administrativas de la Universidad Antonio Nariño, por haber aceptado el diálogo sobre estos temas con la revista Papeles. Presentamos las preguntas hechas y luego las respuestas de cada uno de los invitados.

1. ¿Puede usted mencionar los tres autores con mayor influencia sobre su vida como economista?

2. ¿Cuál es el mayor cambio entre los tiempos de estudiante y los tiempos de profesor o profesional de la economía?

3. ¿Cuál es la mayor crítica a las facultades y a los profesores de economía de nuestro tiempo?

4. ¿Qué es lo más inútil en la enseñanza de la economía que usted ha percibido?

5. ¿Qué cambiaría en la enseñanza de la economía?

6. ¿Cómo cree usted que aportaría la ciencia económica al diseño de una mejor educación en Colombia?

7. ¿Cree usted que las facultades de educación en Colombia deberían tener un mayor componente en fundamentos de teoría económica en relación con las posibilidades en el mercado del capital humano en formación?

\section{Dr. Erick Behar Villegas}

Decano de la Facultad de Ciencias Administrativas, Económicas y Contables, economista de la Universidad de Bochum, Alemania, con enfoque en econometría y economía internacional.

1. Menciono 5, porque siento que realmente me han influenciado todos por igual, sin ranking: Joseph Alois Schumpeter, Richard Thaler, Steven Levitt, Mariana Mazzucato, Seth Davidowitz,

2. Estudié economía en Alemania entre 2007 y 2010, es decir, hace casi unos 10 años. El cambio más grande se viene dando desde lo

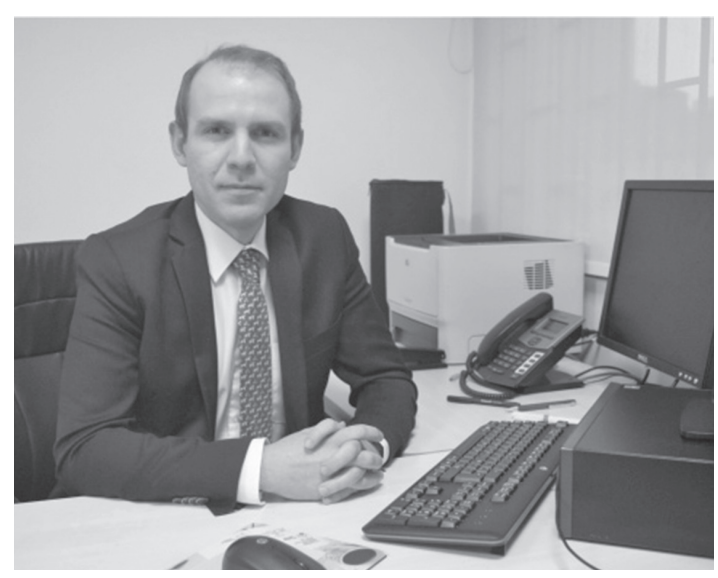
interdisciplinario, porque la economía no se 
puede aislar de otras disciplinas. Un cambio profundo para mí es no haber tenido en cuenta en aquella época la psicología, cuando hoy es un fundamento en mi percepción de la economía, inclusive en temas de macro.

3. No podemos matematizarlo todo, y eso está sucediendo. También se debe dar un espacio al vínculo con la historia, sin olvidar que necesitamos las matemáticas. Algo que critico es la aproximación clásica a la microeconomía, sin incluir temas complejos como la psicología en las perspectivas de los consumidores. Para mí, la enseñanza de la microeconomía debe cambiar profundamente, no solo en Colombia, sino también en Europa y EE.UU. Otro tema grave es aislarnos como economistas y no aprender de disciplinas hermanas, creando ecosistemas interdisciplinarios desde la misma universidad.

4. Lo más inútil en la economía son las ideologías. Debemos enseñar a pensar, contrastar, criticar, evaluar, medir, entre otros, pero no podemos hacerlo desde una ideología, sino desde un acervo de corrientes de pensamiento. Algo en lo que no debemos perder mucho tiempo es en clases de derecho. Se debe enseñar lo fundamental, lo básico, la casuística, pero no perdernos en un sistema ineficiente que ha hecho tanto daño a Colombia. Solo la regulación que nos ayude a entender el sistema es clave, para tener economistas dispuestos a cambiarlo.
5. Haría obligatorios los proyectos interdisciplinarios, el estudio de temas de psicología y dedicaría un buen tiempo a tres temas clave:

- Informática (enseñar a programar, manejar bases de datos SQL, etc.)

- Hacer proyectos de interacción real con el entorno desde los problemas reales

- Aprendizaje de otros idiomas

6. Tenemos todo el potencial de promover lo interdisciplinario y promover la calidad. Esto supone formar economistas que tengan competencias matemáticas, informáticas, entre otras; además, una formación cívica que vaya más allá de las ideologías. Necesitamos formar economistas que (si así lo quieren) se entiendan como verdaderos pensadores y ejecutores de lo público desde la eficiencia, no desde la burocracia. Esto no es pensable sin laboratorios de innovación gubernamental y un fuerte énfasis en economía experimental.

7. Absolutamente, pero depende de lo que entendamos por teoría económica. $\mathrm{Si}$ hablamos de meros y abstractos conceptos, no. Pero si hablamos de temas como comprensión, medición, aplicación de variables y conceptos de macroeconomía, instrumentos de política monetaria y fiscal, conocimiento de complejidades del sistema financiero, conocimiento de psicología del consumidor y un refuerzo en estadística, sin duda.

\section{Dr. Mauricio Pérez Salazar}

Estuvo al frente de la decanatura de la Facultad de Economía de la Universidad Externado por muchos años; economista de Princeton University.

1. Adam Smith, John Stuart Mill, Arthur Lewis.

2. Creo que el paso de ser estudiante al ejercicio profesional está marcado por algo que no se adquiere fácilmente en el aula: el sentido de lo práctico.

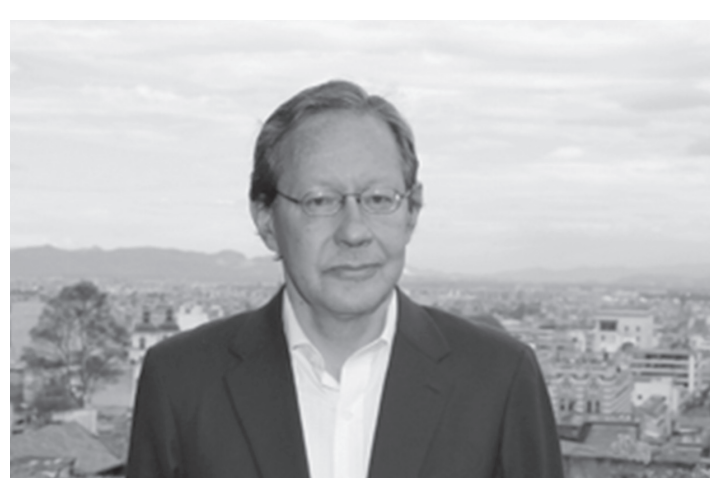


3. Cualquier generalización es aventurada. Cada facultad, cada profesor tiene sus fortalezas y debilidades. Pero todos enfrentan el mismo reto: desarrollar en sus estudiantes la capacidad del pensamiento crítico.

4. De nuevo, creo que generalizar es atrevido. En mi experiencia, distintos estudiantes tienen diferentes aptitudes e inclinaciones. Lo que resulta inútil para uno puede ser utilísimo para otro.

5. El modelo colombiano de enseñanza de la economía tiene un vacío: pocas humanidades. Considero que saber algo de eso hace mejores economistas.

6. Hay un aporte instrumental; por ejemplo, la medición de la calidad educativa, la indagación sobre sus causas, el diseño de mejores políticas públicas para el sector. En cuanto a contenidos, todo educando en cualquier disciplina debería tener en su bagaje intelectual dos conceptos claves de la economía: la restricción de la escasez y el costo de oportunidad.

7. Los fundamentos de la teoría económica son el sine qua non. Todo lo demás es un añadido. Si no se tiene un buen dominio de los fundamentos no se puede ser un buen economista. Y por supuesto, si una facultad de economía en Colombia, o en cualquier parte, no logra impartirlos a sus estudiantes no está cumpliendo con su responsabilidad con ellos y con la sociedad.

\section{Dra. Juanita Villaveces Niño}

Directora de la Escuela de Economía en la Universidad Nacional. Es economista de la Universidad Nacional, con maestría en Política Comparada del London Schools of Economics y Doctora en Estudios Políticos de la Universidad Externado de Colombia.

1. Puede sonar cliché, pero Adam Smith ha sido un autor que ha logrado influenciar mi forma de entender el análisis del funcionamiento de una sociedad. Si bien la obra más conocida para los economistas es La Riqueza de las Naciones, Smith fue un autor integral que exploró al sujeto más allá del mercado. Su trabajo la Teoría de los Sentimientos Morales reconoce la simpatía y la reciprocidad como motores en las decisiones; así como en la obra de Lecciones de Jurisprudencia resalta la necesidad de reglas y limitantes o, una mano visible en la sociedad.

Douglass North. Descubrí a North en mis cursos con el profesor Jesús Antonio Bejarano. Leer a North tiene entonces una doble connotación: recordar al "profe" Bejarano y su curiosidad y revelar una forma de

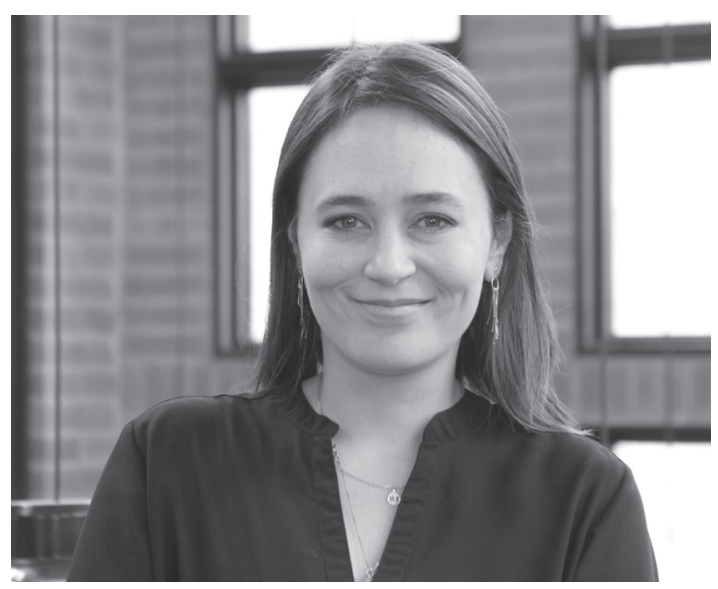

entender la economía desde los procesos históricos y las tensiones sociales. Su idea de instituciones marca mi enfoque económico.

Elinor Ostrom. No es economista, pero me ayudó a entender que las decisiones no se circunscriben a lo individual, sino que, socialmente, también se forman decisiones y que hay temas sensibles que vinculan a varios actores dispuestos a cooperar. Esta autora coincide con mi interés genuino por comprender la política para continuar entendiendo la economía. 
2. Sin lugar a dudas, el acceso a información y el manejo o relación con la misma. Estudié con la sutil llegada de internet a Colombia que, básicamente, servía para el envío de correos pero no para acceder a cúmulos de información. Al finalizar las clases, corríamos rumbo a la biblioteca para tener acceso a los libros, so pena de que algún compañero egoísta retirara primero el libro y lo "escondiera" durante los días de préstamo. El acceso tan reducido a libros y datos nos llevaba a leer con entusiasmo lo que encontráramos asociado, quizá de un profesor que nos prestaba sus libros o por sorpresa en las largas jornadas en la BLAA. Hoy veo a estudiantes más conformes por la certeza de encontrar información a la mano. Conformes y menos curiosos; $y$, de hecho, mucho menos interesados en leer la información que pueden encontrar. Quizá es parte de un nuevo aprendizaje que lleva poco tiempo aún.

3. Copiar formas de enseñanza, de temas, de literatura y de evaluación según lo que hay en las universidades extranjeras de renombre. Copiar sin reflexionar sobre cómo llevarle ese conocimiento y prácticas a estudiantes que viven realidades distintas y, seguramente, tienen preguntas diferentes. Falta originalidad de calidad.

4. No creo que haya temas inútiles. Más bien, metodologías inútiles. Siendo estudiante, sentía que la econometría era inútil, pero creo que tuve un mal profesor con una mala

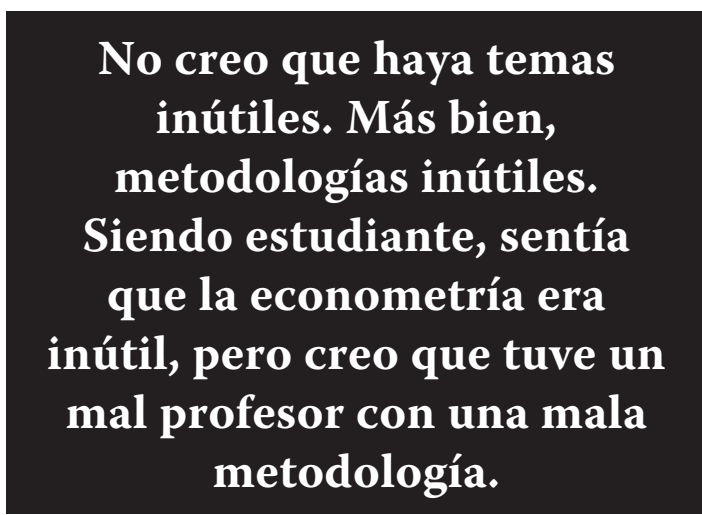

metodología. Nunca me explicó para qué servía la metodología. Creo que lo inútil tiene que ver con la incapacidad de darle un contexto a las cosas o presentar la relevancia más allá de la obligación o la nota.

5. nseñanza basada en realidades. En contextos propios y preguntas que sociedades y economías como la nuestra se hacen. Hay iniciativas en esa perspectiva que pueden ayudar a la enseñanza. En Colombia hay profesores innovadores en prácticas docentes en economía, pero no son tomados en cuenta porque se privilegian los títulos sobre la pedagogía. Es decir, la reflexión sobre ser buen profesor no ha permeado la economía en su totalidad.

6. Reflexionando sobre el papel de la docencia y llevando la discusión a ver al menos tres componentes de un buen docente: la formación, la investigación y la habilidad para conectarse con distintos grupos y distintas generaciones. En principio, los dos primeros son exigencias a la hora de entrar a ser parte de una universidad. El tercero, la habilidad de conectarse con otros, hace parte de cómo hablamos, cómo usamos el lenguaje para expresar, pero no solo el verbal y escrito, también el corporal y el satírico. Esa discusión me gusta, la he hecho desde hace unos diez años cuando entendí que el humor debe ser parte de la clase y lo abordé usando caricaturas en un curso.

7. No. Creo que las facultades de educación están haciendo un papel importante en entender y ayudar a otros a comprender la enseñanza en un sentido amplio. Llamarlas a conocer un poco de todas las disciplinas sería un error en su razón de ser. Creo, más bien, que su rol es continuar ampliando el mercado a los profesores de distintas disciplinas. Es decir, todos necesitamos de las facultades de educación para repensar nuestro papel de docente que, por definición, no es estático en el tiempo y debe ser capaz de transformarse según las necesidades. 


\section{Dr. Carlos Julio Martínez Becerra}

Economista de la Universidad Nacional. Diploma de Estudios avanzados (Ms) y Doctorado en Economía y Sociedad - sistemas comparados- de la Universidad de París XNanterre. Estadías postdoctorales en Economía Marítima y en Geopolítica de Estados Unidos de la EHESS de París. Profesor-investigador con publicaciones en integración económica, geopolítica de la energía, seguridad y defensa. Invitado de varias universidades como Harvard, UNAM, U. de París, entre otras. Actual decano nacional de la Facultad de Ciencias Económicas y Administrativas de la UAN.

1. John Maynard Keynes, Antonio García Nossa, Thomas Piketty.

2. Conocer en el ámbito de la economía real los postulados teóricos, pues casi siempre son superados en la vida real. Esto es muy importante, porque se descubren hipótesis que inducen conductas que no corresponden con la realidad.

3. La mayor crítica es hacer abstracción de la realidad económica en nombre de la pureza intelectual, como un recurso para no comprometerse con las necesidades de la sociedad. No olvidar que el tecnócrata casi siempre es un ideólogo que actúa sin tener formación plena como ciudadano.

4. Sería muy importante interactuar con los agentes económicos individuales y colectivos para humanizar y enriquecer las teorías económicas en sus diversas aplicaciones públicas, privadas y de economía solidaria.

5. Retomar el oikos para hacer un balance entre teoría y política de manera que las especulaciones cuantitativas puedan alimentarse de reflexiones socioantropológicas que interpreten de nuevo al ciudadano con sus derechos y deberes y no solo como un consumidor que actúa por influencias cada

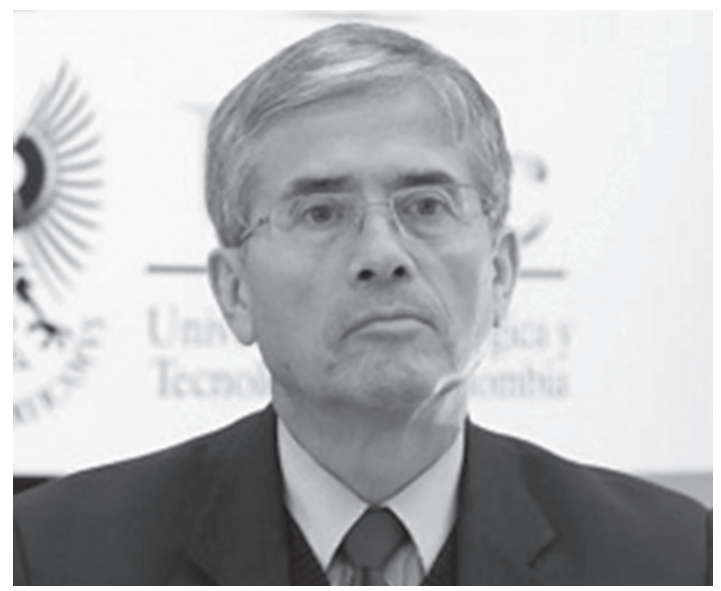

vez más derivadas de patrones de consumo inducidas por las redes sociales. Evitar el efecto manada con buena información.

6. La consigna de "aprender haciendo" puede conducir a un mejor aporte de la cultura económica para comprender el reto del crecimiento económico; pero también el tema de la distribución de la riqueza derivada. La economía aplicada empieza por conocer el entorno y tener visión global para dar valor a las tecnologías tradicionales, sin desconocer tecnologías intermedias y de punta.

7. Los fundamentos de economía deben hacer parte de la cultura ciudadana. Especialmente si se tiene en cuenta que la economía mundial está enviando señales de la nueva competencia en economía basada en ventajas tecnológicas y del conocimiento más que en esquemas comerciales de consumo con endeudamiento. El bienestar no puede depender de procesos de adaptación tecnológica (consumidores tecnológicos), sino de la creación de conocimiento que derive en invenciones e innovaciones tecnológicas para generar valor agregado a los recursos locales y regionales, siempre con visión universal. 